\title{
Author Correction: Tuning of the Berry curvature in 2D perovskite polaritons
}

Laura Polimeno, Giovanni Lerario, Milena De Giorgi (1D, Luisa De Marco, Lorenzo Dominici, Francesco Todisco, Annalisa Coriolano, Vincenzo Ardizzone, Marco Pugliese (D), Carmela T. Prontera, Vincenzo Maiorano, Anna Moliterni,

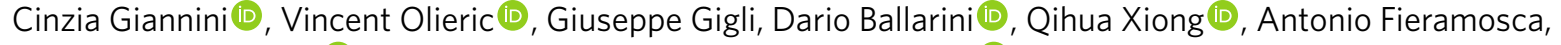
Dmitry D. Solnyshkov B, Guillaume Malpuech and Daniele Sanvitto (D)

Correction to: Nature Nanotechnology https://doi.org/10.1038/s41565-021-00977-2, published online 21 October 2021.

In the version of Article initially published, there were omissions in the list of corresponding authors. In addition to Milena De Giorgi, Luisa De Marco (luisa.demarco@nanotec.cnr.it) and Dmitry D. Solnyshkov (dmitry.solnyshkov@uca.fr) should have been indicated on the title page and correspondence and request for materials line as additional corresponding authors.

The errors have been corrected in the online version of the article.

Published online: 12 November 2021

https://doi.org/10.1038/s41565-021-01046-4

(๑) The Author(s), under exclusive licence to Springer Nature Limited 2021 